\title{
H. P. Meinzer
}

German Cancer Research Center Department of Medical and Biological Informatics

Heidelberg, Germany

This synopsis concerns some recent publications and trends in image and signal processing in the field of medical informatics. This particular field is mainly application-oriented with technical applications relevant to health and health care. A number of papers were proposed by IMIA Societies and others and individually reviewed by three referees. This review process resulted in a selection of seven publications originally printed in seven specialized medical and technical journals.

Four of the papers fall into the category of image processing, and three deal with signal processing. The image processing papers are on PACS architecture (picture archiving and communication system), blood smear analysis, lung nodule diagnosis in Röntgen images, and an attempt of accoustic interpretation of images. The signal processing papers address the interpretation of electro-encephalograms (EEG), the analysis of electrocardiograms (ECG), and a reference database of ECGs. This selection represents topics of actual international research, the authors coming from around the globe and working in European, American, and Asian laboratories.

The first paper to be discussed is by Huang and Taira: Infrastructure design of a picture archiving and communication system [1]. It is a review article on PACS architectures. One

\section{Synopsis}

\section{Image and Signal Processing}

should assume that the infrastructure point of view is self-evident for PACS design; unfortunately, this was not always true. The authors suggest a PACS modularization emphasing connectivity. The components are acquisition computers, cluster controllers, database servers, display stations, and an all-connecting network. This infrastructure covers firstly the collection of data from the primary image producing devices. Today, the different machines have only begun to use image format standards, e.g., ACRNEMA or SPI. The data must then be shipped in a network from device to device and stored in a database. The data must also be retrieved, processed and presented to the users. These facts seem simple. However, although the first PACSs did fulfil these obvious tasks, the systems were closed boxes and unable to communicate. The computer tomographs (CT), the magnetic resonance imaging (MRI) as well as PET and SPECT systems were always digital techniques. X-ray and ultrasound images still live a digitally isolated life on isolated equipment and are available as photographic images. Today, an integration process is on its way, making available the complete digital information of all patient images. The first PACSs suffered not only from missing exchange standards, but also from a number of planning and technical shortcomings. The resolution of the images was low, and yet the amount of data produced per day (or per patient) was too large for the hardware components. The space in memory and the magnetic storage devices was small and expensive. The connecting networks were too slow and the high-resolution displays expensive. All these drawbacks have been overcome in the last years. The contribution of Huang and Taira [1] shows a way out of the design problems with suggestions to overcome the 'closed architecture' problems of the early years of PACS. Still missing is a flexible standard for image formats since, for example, ACR-NEMAlacks the ability to store image sequences and suffers from inflexible data types for a number of representations.

Poon et al. published Automated image detection and segmentation in blood smears [2]. The aim of the contribution is an automated diagnosis and classification of leukemias. They claim that their method differs from others in the simplicity of the algorithm and the evaluation of both touching as well as non-touching cells in the image. Their method involves spectral images, an algorithm to separate touching cells, segmentation of cells into nuclear and cytoplasmic regions and an analysis of the segmented regions. A large part of the paper describes the advantage of including touching cells in the analysis. The identification and separation of touching cells is done in a straightforward way with cell-boundary analysis allowing for deliberate partitioning of a 'super cell' into two. The problem of 
overlapping cells (more than just touching) remains, and with it the danger of false-negative results as the system cannot distinguish abnormal cells from massively overlapping ones. The problem of false-positive results is approached by a set of image preand postprocessing routines. It is considered less problematic since the positive slides are manually processed by pathologists anyway.

For over 20 years, attempts have been made to automate smear images and chromosome analysis, the two areas suffering from the same problems. Today there are still no solutions available that are robust in routine applications. This does not reflect a lack of talent of the researchers involved, but a general shortcoming of computer-based image analysis. Human interpretation of images is still not fully understood. We know that a human observer perceives an image qualitatively, the 'Gestalt'-oriented view. Since a computer sees pixels and perhaps some neighboring pixels, it cannot recognize the image because it does not see the whole. The gap from pixel-oriented analysis (the signal) to the global view and symbolic description remains. The actual research tries to bridge the gap by inclusion of aprioriknowledge, artificial intelligence approaches, and neural nets concepts, e.g., topological feature maps as suggested by Kohonen [3]. We must admit that much work remains to be done. The small advances in computer-based image understanding should not discourage us. Perhaps, a better understanding of the human ability to interpret images may show the way to practical and robust systems.

Problems similar to the ones mentioned previously are found in the contribution of Matsumoto et al.: Image feature analysis of false-positive diagnoses by automated detection of lung nodules [4]. The team wants to support the diagnosis of lung cancer by automated analysis of chest radiographs. Lung cancer is accompanied by visible lung nodules. Radiologists can fail to diagnose pulmonary nodules in as many as $30 \%$ of positive cases, but $90 \%$ of peripheral lung cancers were visible, in retrospect, on previous films. An earlier computer program of the authors detected too many nodules. In this paper, they attempt to reduce the number of false-positive diagnoses by enhanced programs and the inclusion of knowledge from experienced chest radiologists.

The specific sources of false-positive detections are quite obvious. The computer falsely identifies as nodules aggregates of vessels, rib-rib (or clavicle) crossings and rib-vessel crossings. The main weapons in the detection of pseudo nodules are the analyses of the circularity and the irregularity of the nodule candidates. The improved program produced a reduction of false-positive nodules from 12 to 5 per image. This improvement is of limited success since too many wrong nodules still remain. Perhaps the most important feature is that none of the positive nodules was suppressed. As mentioned above, an improvement of the image analysis was achieved by the inclusion of medical knowledge, the sad point being that this knowledge is not expressed in symbolic form in an approachable knowledge database but incorporated in the source code in an unaccessible hidden manner. This prevents updating and minor changes if better understanding of the human medical diagnosis processes becomes available.

The last selected paper in the image processing section is by Meijer: An experimental system for auditory image representations [5]. The ultimate goal of this work is a system for the visually impaired or blind, i.e., a sys- tem that could sing an image to man That sounds at first fantastic, but per-1 haps images can be translated into sound. Considering the massive handicap of a blind person, anything that can be of help must be considered There have been attempts in this direc. tion, attempts that tried to transform images into physical impulses on the sensors of the body, e.g., on the back This region is large but unfortunatef of very low spatial resolution. Alson the mechanical devices would not be very handy. The basic idea of Meijet can be explained quite easily. The grey values in the rows of a digital image matrix are translated into inten. sities of individual frequencies assigned to each row. The image columns are then sung one by one, translating the rows in the horizontal direction of images sequentially as a function of time. The resulting sound is very hard to imagine, and it remains unclear how the ears perceive this sound package representing an image, The author admits that the system still awaits a thorough evaluation with blind persons. Nevertheless, technical feasibility has been established.

The three selected contributions in the field of signal analysis include the one by Pfurtscheller et al.: Prediction of the side of hand movements from single-trial multi-channel EEG data using neural networks [6]. My first comment here is that I disfavor titles that are as long as abstracts, a very popular procedure in our days. The authors recorded 30 channels of EEG data prior to voluntary right or left hand movements. Event-related desynchronization (ERD) was quantified in the $8-10 \mathrm{~Hz}$ and $10-12 \mathrm{~Hz}$ bands in single trial data and used as training input for a neural network. After a training period, the network was able to predict the side of hand movement from EEG data recorded prior to the onset of movement. On the scalp, 30 electrodes collect data that 
are first used to train a topological feature map, which is a class of neural network becoming increasingly popular. The spatio-temporal patterns are then used to predict rhythmic activities. The ultimate goal of the development would be a direct input device, for example the brain control of a robotic arm or hand. Scalp electrodes are noninvasive, the remaining question being how much information can be drawn from such a device. The decision whether the coming movement involves the left or the right hand is binary. It is unclear if there is more information derivable from a few electrodes. Direct implantion of electrodes can be the next step, but for this too little is still known about the detailed regions of the brain responsible for specific action. The important contribution of this paper, however, is to show in which direction scientists today direct their interest and research. The dream of a direct brain-computer interface is, of course, fascinating and every large advance is started by small steps. The use of Kohonen's topological map [3] is very interesting. The advantage of it is the preservation of the topology of the feature space, the self-adjusting learning process and the open number of features. It seems as if the Kohonen map has a bright future and continues to earn a wider appreciation.

Kors et al. wrote Variability in ECG computer interpretation [7]. The variability of the electrocardiogram can be due to extrinsic noise or intrinsic factors, such as changes in the volume conductivity. This varability makes the automatic analysis of ECGs complicated and less reliable. The classic approach, therefore, is the sampling and averaging of a series of complexes, thus creating a, hopefully, representa(ive PQRST complex. The authors suggest an alternative method: the derivation of a set of measurements trom each complex (signal) in a re- cording, a subsequent individual classification and a final classification, combined from the individual ones. The total accuracy of the combined classifications of the individual complexes proved to be slightly higher than that of the classifications of the averaged complexes. This overcomes the problem of the intrinsic varability.

The European ST-T database: standard for evaluating systems for the analysis of ST-T changes in ambulatory electrocardiography was published by a large European team headed by Taddei [8]. The goal was an ECG database for assessing the quality of ambulatory ECG (AECG) monitoring systems. Thirteen research groups from eight countries provided AECG tapes. The annotation scheme was revised to be consistent with the Bostonian MIT$\mathrm{BIH}$ arrythmia database and the American Heart Association formats. There is convincing evidence that STsegment shifts of the ischemic types can be detected by AECG. Recurrent ischemia, in particular, most of which is silent and occurs only during daily life at low activity and heart rate levels, may be detected by AECG. The last two statements are found in a report written by a joint committee of the AHA and the American College of Cardiology. They indicate the importance of a standardized, reliable teaching and learning set of AECG data. The European ST-T database is intended to be used mainly for evaluating the performance of algorithms which detect changes in ST segments and T-wave morphology. Combined with the American databases, the quality of AECG instruments can be assessed.

The wide range of the seven articles of image and signal processing in medical applications shows where we are and where we are going. It shows fascinating dreams and visions to be pursued in the future as well as solid basic work on standards and integration of diverse components directed to the ultimate goal of enhanced medical care. I consider it a great development that European researchers start to cooperate, as they have been doing for a long time with their American partners. The international partnerships should be further extended and encouraged in general. This international community of scientists should use their privilege of global connections across the borders for a further integration and cooperation in this world. We only have this one and we are responsible for it.

\section{References}

[1] Huang HK and Taira RK. Infrastructure design of a picture archiving and communication system. Ajr Am J Roentgenol 1992;158:743-9.

[2] Poon SSS, Ward RK and Palcic B. Automated image detection and segmentation in blood smears. Cytometry 1992;13:766-74.

[3] Kohnen T. Self-organization and Associative Memory (3rd ed). Berlin: Springer, 1988

[4] Matsumoto T, Yoshimura H, Doi K, Giger ML, Kano A, MacMahon H, Abe $\mathrm{K}$ and Montner SM. Image feature analysis of false-positive diagnoses produced by automated detection of lung nodules. Invest Radiol 1992;27:587-97.

[5] MeijerPBL. An experimental system for auditory image representations. Ieee Trans Biomed Eng 1992;39:112-21.

[6] Pfurtscheller G, Flotzinger D, Mohl W and Peltoranta M. Prediction of the side of hand movements from single-trial multi-channel EEG data using neural networks. Electroencephalogr Clin Neurophysiol 1992;82:313-5.

[7] Kors JA, van Herpen $G$ and van Bemmel $\mathrm{JH}$. Variability in ECG computer interpretation. Analysis of individual complexes vs analysis of a representative complex. J Electrocardiol 1992;25:263-71.

[8] Taddei A, Distante G, Emdin M, Pisani P, Moody GB, Zeelenberg C and Marchesi C. The European ST-T database: standard for evaluating systems for the analysis of ST-T changes in ambulatory electrocardiography. Eur Heart J 1992;13:1164-72. 